Military Technical College Kobry El-Kobbah, Cairo, Egypt

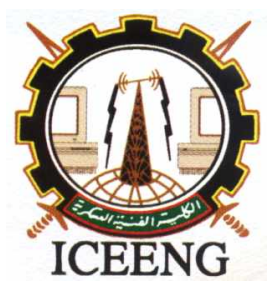

\author{
$6^{\text {th }}$ International Conference \\ on Electrical Engineering \\ ICEENG 2008
}

\title{
Active impedances of elements in pencil beam and fan beam planar finite phased arrays of dipoles with low side lobe level
}

$$
\text { By }
$$
D.F. El-Hossary *
E. A. El-Diwany **
H. H. El-Tamaly ***

\section{Abstract:}

The active impedances of the elements of planar finite phased arrays of dipoles are investigated for excitations which produce either pencil beam or fan beam radiation patterns with low side-lobe levels produced by tapered excitations. It is found that with tapered excitations the elements impedances in the array change considerably with beam scanning when compared with the case of uniform excitation.

\section{Keywords:}

Phased antenna arrays, planar dipole arrays, scan active impedance, tapered excitation (pedestal, Taylor $\bar{n}$ ), pencil beam and fan beam (cosec)

* $\quad$ Brig. Gen. (R) Dr., Egyptian Armed Forces.

** Assoc.Prof. Dr., Electronics research institute.

*** Prof. Dr., Faculty of Engineering, Minia University. 


\section{Introduction:}

The active impedances of the different elements of a finite antenna array are important for impedance matching. These impedances change with beam scanning in the different planes and with the location of the element in the array. H-plane scan resistance and scan reactance increase with scan angle, while they decrease in E-plane [1]. The impedances of the elements and the current distribution on finite arrays show the presence of an interference pattern between two types of waves [1, 2, 3]. The first one is the radiated wave by the antenna with its scan angle. The second one is a traveling wave on the array surface, which could be a surface wave or a leaky wave $[1,2,3,4,5]$. For large arrays the elements impedances far from the array edges are nearly the same, thus this impedance is obtained from the solution of the infinite array problem which reduces to the solution of a single element problem. However, in order to find the elements impedances near the edges, one needs to invert the impedance matrix relating the currents and voltages in the array. For large arrays the matrix is huge, thus the elements impedances near an edge may alternatively be obtained by studying an array which is infinite in one direction and finite in the other direction in which scanning is performed $[1,2]$. The periodicity in the infinite direction makes the elements active impedances in this direction to be similar, thus the problem reduces to finding the impedances only along the finite direction which leads to considerable reduction in the matrix inversion time. The direction with finite number of elements can be in the E- or the $\mathrm{H}$ - plane. However, this solution does not provide correct results for the elements near the corners. The published results for large arrays are mainly for finite by infinite arrays and uniform excitation of the array.

This paper investigates planar finite phased arrays of cylindrical dipoles in free space, which may be backed by a ground plane to produce a beam in only one side of the ground plane. Emphasis is given to the study of the active impedances of the different elements of the antenna array with beam scanning in both the E- and $\mathrm{H}$ planes, including the impedances of the elements at the edges and corners of the finite array. The excitations used in the array produce either pencil beam for tracking radars or fan beam for search radars (cosecant pattern). The current distributions considered include

a uniform distribution which is characterized by a narrow beam and high gain, and tapered distributions from the array center to the edges (pedestal and Taylor $\bar{n}$ ) to produce low sidelobe levels, which have a main advantage in avoiding jamming through the sidelobes. The effects of the tapered excitations on elements impedances and traveling leaky wave are investigated. 


\section{Analysis of the array dipole impedances}

Let us consider a finite array of $\mathrm{M} \mathrm{X} \mathrm{N} \mathrm{dipole} \mathrm{elements} \mathrm{at} \mathrm{height} \mathrm{'} \mathrm{h}$ ' above a ground plane as shown in Fig. 1.

The feed network for each dipole consists of a voltage source in series with a source impedance $Z_{\mathrm{s}}$. The equations for the applied voltages for the dipoles in the array can be written in terms of the array currents, self, mutual impedances $Z_{\mathrm{ij}}$ and source impedances $\mathrm{Z}_{\mathrm{s}}$ as $[1,2]$ :

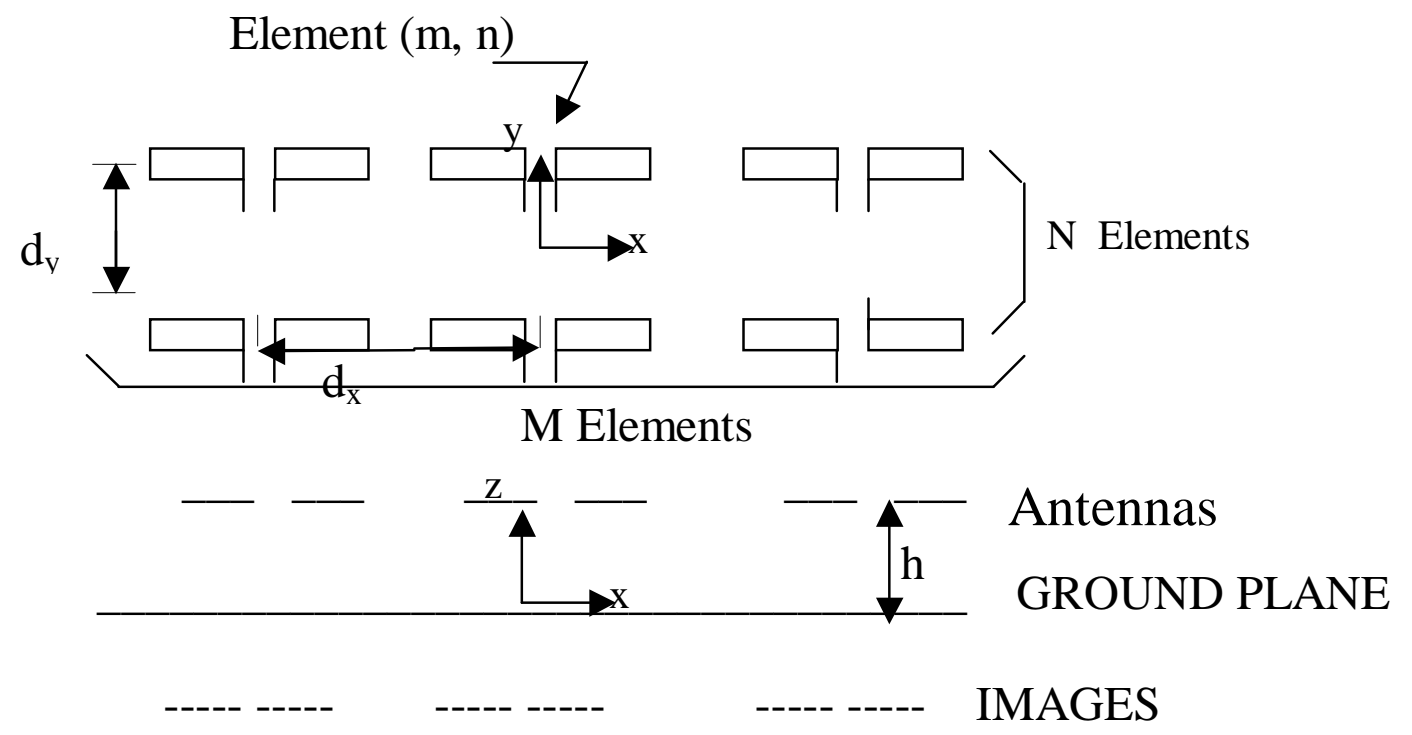

Fig. $1 \mathrm{Mx} \mathrm{N}$ dipole elements at height ' $h$ ' above a ground plane

$$
\begin{aligned}
& V_{1}=\left(Z_{11}+Z_{S}\right) I_{1}+Z_{12} I_{2}+--------+Z_{1 n} I_{n} \\
& V_{2}=Z_{21} I_{1}+\left(Z_{22}+Z_{s}\right) I_{2}+--------+Z_{2 n} I_{n} \\
& V_{n}=Z_{n 1} I_{1}+Z_{n 2} I_{2}+--------+\left(Z_{n n}+Z_{s}\right) I_{n}
\end{aligned}
$$

The phase of the driving voltage in the $m^{\text {th }} n^{\text {th }}$ element in the array is expressed in terms of the scan angles $\Theta_{0}$ and $\varphi_{0}$ as:

$$
V_{m n}=A_{m n} \exp \left(-j \alpha_{0} k x_{m}-j \beta_{0} k y_{n}\right) \text {, }
$$

where

$\alpha_{0}=\sin \theta_{0} \cos \phi_{0}, \quad \beta_{0}=\sin \theta_{0} \sin \phi_{0}, \quad k=2 \pi / \lambda, \quad \lambda$ is the wavelength,

$\left(x_{m}, y_{n}\right)$ position of the $(\mathrm{m}, \mathrm{n})$ element, $x_{m}=m d_{x}$, and $y_{n}=n d_{y}$

Any tapered or non uniform excitation is included in the amplitudes $\mathrm{A}_{\mathrm{mn}}$.

The array element currents are the solution of this matrix equation. From the current vector the active scan impedance of each element in the array is determined by 
subtracting the source impedance from the total element impedance obtained by dividing the source voltage by the current. For maximum power transfer, the source impedance is taken as the conjugate of the center element impedance at broadside scanning (which is nearly equal to that in an infinite array).

\section{Mutual impedance between two cylindrical dipoles and the used source impedance} Consider two center-fed dipoles in free space having identical lengths and radii (2l, c). The current on either dipole has approximately a sinusoidal distribution, namely for dipole 2;

$I_{2}\left(\zeta_{2}\right)=I_{m 2} \sin k\left(l_{2}-\left|\zeta_{2}\right|\right)$.

The vertical component of field at dipole 2 due to dipole 1 can be written as [6].

$$
E_{z}=-j 30 I_{m}\left(\frac{e^{-j k r_{1}}}{r_{1}}+\frac{e^{-j k r_{2}}}{r_{2}}-2 \cos k l_{1} \frac{e^{-j k r}}{r}\right)
$$

where the distances $\mathrm{r}, \mathrm{r} 1, \mathrm{r} 2$ are from the observation point on dipole 2 to the center and the ends of dipole 1, respectively. The mutual impedance between the two dipoles is given by [6]

$\mathrm{Z}_{21}=\frac{1}{\sin k l_{1} \sin k l_{2}} \int_{-l 2}^{12} \frac{E_{z}\left(\zeta_{2}\right)}{I_{m_{1}}} \sin k\left(l_{2}-\left|\zeta_{2}\right|\right) d \zeta_{2}$

Substituting Eqn. (3) in Eqn. (4) gives the expression for the mutual impedance.

In the presence of a ground plane, Fig.1, a term similar to $E_{z}$ is subtracted from it in which the distances are from the image source dipole behind the ground plane to dipole 2 (the image of a current parallel to the ground plane has a negative sign). The self impedance can be computed by considering the mutual impedance between coincident dipoles, where the current and field are considered to be on the dipole periphery and axis, respectively. In order to find the matched source impedance for the finite array, it is set to be the conjugate of the active impedance in the infinite array with zero scan angle. For a flat dipole of length ' $a$ ' and width ' $b$ ' (equal to 4 times the radius of the equivalent cylindrical dipole [7]) this impedance is given by [8, 4],

$$
Z_{P}=\frac{1}{d_{x} d_{y} k^{2}} \sum_{m} \sum_{n} \bar{Z}\left(\frac{\lambda}{d_{x}} m^{\prime}, \frac{\lambda}{d_{y}} n^{\prime}\right)
$$

where

$$
\begin{aligned}
& \bar{Z}(\alpha, \beta)=960 \pi\left(\frac{a}{\lambda}\right)^{2} F^{2} \cdot G^{2} \cdot H \cdot R \\
& G=\frac{\cos \left(\frac{a k}{2} \alpha\right)}{1-\left(\frac{\alpha k a}{\pi}\right)^{2}}, \quad F=\frac{2 \sin (\beta k b / 2)}{\beta k b}, H=\frac{1-\alpha^{2}}{\gamma}, \gamma=\sqrt{1-\alpha^{2}-\beta^{2}}, R=\left[1-e^{-j \gamma 2 k h}\right]
\end{aligned}
$$

Without a ground plane, $\mathrm{R}=1$. 


\section{Voltage distributions used to feed array elements}

The voltage distributions on the array elements used to compute the active dipole impedance, Eqn. (2), are now given.

$\underline{\text { A constant voltage distribution in } \mathrm{x}-\text { and } \mathrm{y} \text { - directions: }}$

$$
V_{m n}=\text { constant }
$$

Non-uniform (pedestal) voltage distribution in $\mathrm{x}$ - and $\mathrm{y}$ - directions [8]:

$$
V_{m n}=V_{0}+V_{1} \cos \left(\frac{\pi m d_{x}}{(M-1) d_{x}}\right) \cos \left(\frac{\pi n d_{y}}{(N-1) d_{y}}\right)
$$

$\mathrm{m}=-(\mathrm{M}-1) / 2$ to $(\mathrm{M}-1) / 2$,

$\mathrm{n}=-(\mathrm{N}-1) / 2$ to $(\mathrm{N}-1) / 2, \quad \mathrm{M}, \mathrm{N}$ odd

The cosine terms drop to zero at the array edges.

Low sidelobe level Taylor $\bar{n}$ distribution in the y-direction

This family of radiation patterns has the first $\bar{n}$ sidelobes nearly at a desired level $\mathrm{R}$ and all sidelobes beyond $\bar{n}$ fall off. The tapered continuous current distribution (here used as exciting voltage) necessary to produce this family can be found e.g. in $[1,4]$

Cosecant pattern in X-z plane

For search radars with fan beam in the vertical plane, it is required that the antenna radiation field pattern should be proportional to the range $R$ and hence to $\operatorname{cosec} \theta$ to illuminate a target at a constant height with a fixed amplitude. The cosecant pattern is constructed from a number of beams pointing at different angles with appropriate amplitudes (Woodward- Lawson method [1, 4]). Each beam results from a uniform current distribution on the array with linear phase distribution. The expressions for the resulting current can be found e.g. in $[1,4]$.

\section{Results and discussion}

The calculated results are given along lines in the array in E- plane (x-direction) and $\mathrm{H}$ plane (y-direction) at scan angles $\theta_{\mathrm{o}}=0^{\circ}$ and $\theta_{\mathrm{o}}=60^{\circ}$. Cylindrical dipole length and radius are $0.486 \lambda$ and $0.002 \lambda$, respectively, which correspond to the resonant length in an infinite array. Element spacing is $0.5 \lambda$ in both $\mathrm{x}$ - and $\mathrm{y}$ - directions. When considering a ground plane, the height above it is $0.25 \lambda$ to enhance radiation in the broadside direction. The number of elements in the array is mainly $51 x 51$.

\section{Uniform excitation in $\mathbf{x}$ - and $\mathbf{y}$ - directions (pencil beam)}

Figures 2 and 3 show active impedances results of [1] for 51 linear infinite array (finite by infinite) and the corresponding calculated results for 51x51 finite array, respectively, in H-plane, at broadside scan $\left(\theta_{o}=0^{\circ}\right)$. The results are seen to be close to each other (the dipole radius in [1] is not given). Figure 4 shows corresponding calculated results with a screen which leads to higher impedance level [6]. Figure 5 shows scan 
impedance results in H-plane at scan angle $\theta_{\mathrm{o}}=60^{\circ}$. It can be noted that with scanning the impedances are not symmetric around the array center, in contrast with the cases with no scanning, $\Theta_{0}=0$. An expected increase in level of scan impedance is seen compared to the level at broadside scanning due to scan in $\mathrm{H}$ - plane. The figure shows

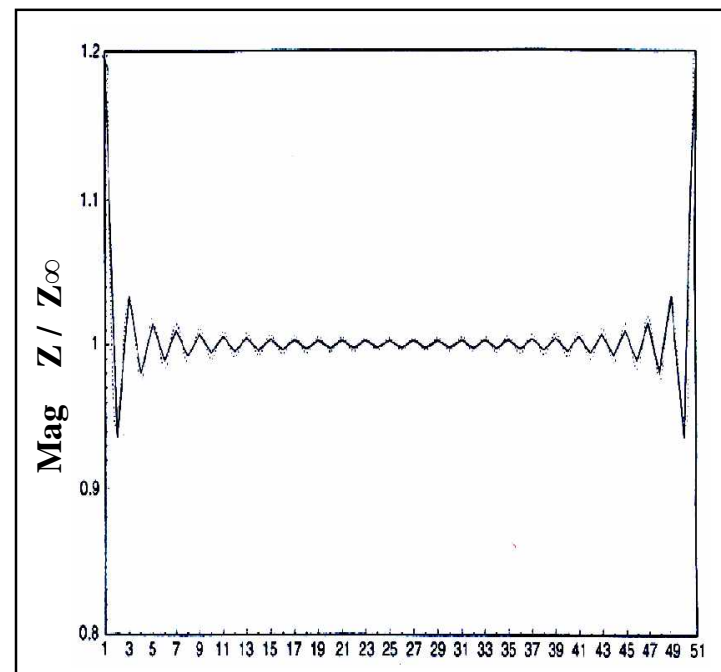

Element number

Fig. 251 linear infinite arrays of resonant dipoles, $\mathrm{d}_{\mathrm{x}}=\mathrm{d}_{\mathrm{y}}=\lambda / 2, \mathrm{H}$ plane, scan at $0^{\circ}$ [1, Fig. 8-22]

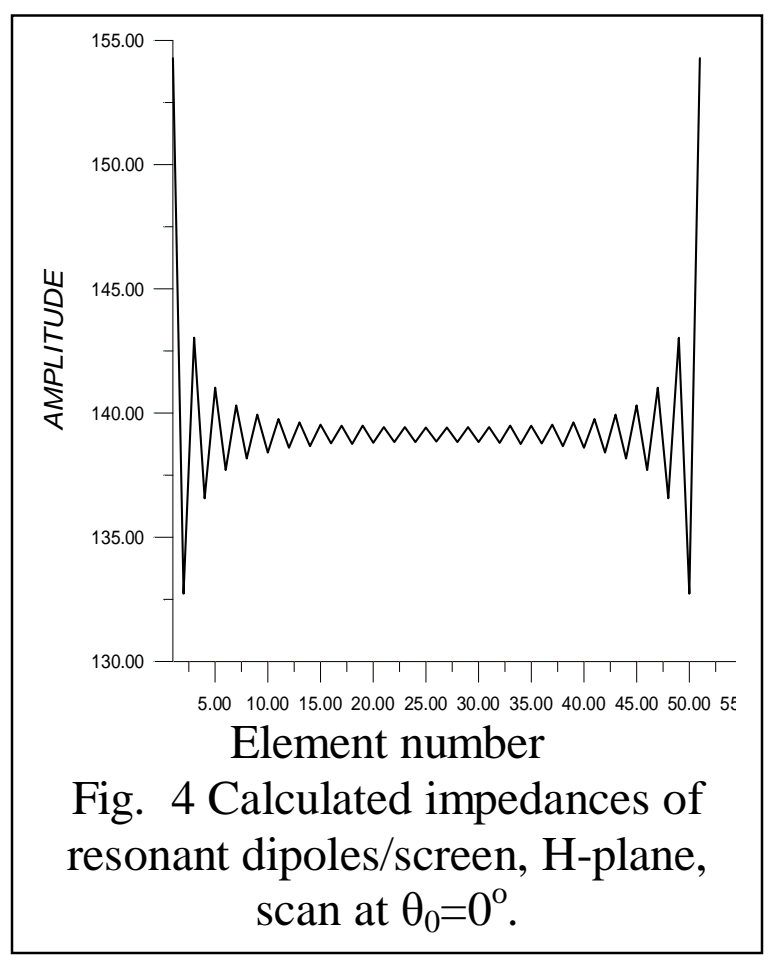

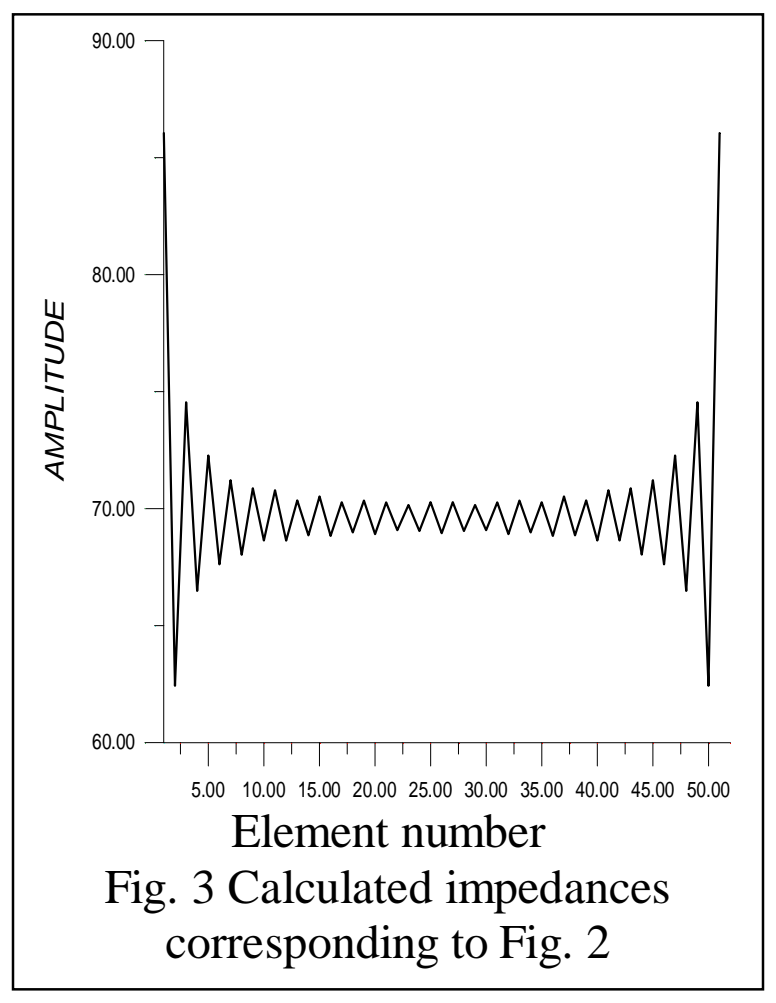

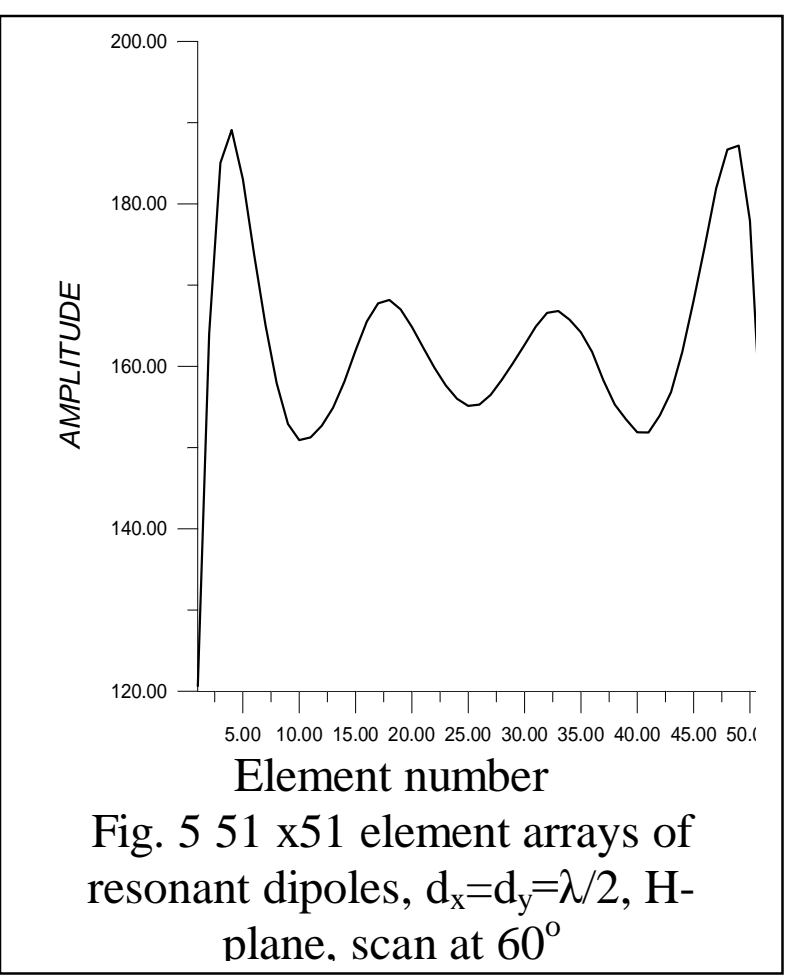


an interference pattern along the array between two waves. The first one is a radiated wave by the antenna at $60^{\circ}$ (propagation constant along array surface, $\beta_{r}=k_{0} \sin \theta$ ). The second one is a traveling wave (leaky wave) having normalized propagation constant $\beta_{\mathrm{t}}$ nearly equal to unity (corresponding to the free space propagation constant $\mathrm{k}$ ) along the array surface. The period of the interference pattern is given by $[1,4,5]$ :

Period in $\lambda=\frac{1}{\beta_{t}-\operatorname{Sin} \theta_{0}}$

Actually, this interference phenomenon exists also in the previous figures, where $\theta_{0}=0^{\circ}$ and also $\beta_{\mathrm{t}}=1$.

Figure 6 shows the elements impedance results at the edge of the array, in H-plane at angle $\theta_{o}=0^{\circ}$. Comparing Fig. 6 with Fig. 3 it is noticed that the level of the impedance at center of the figure changes from $70 \mathrm{Ohm}$ when it passes by the array center to 60 $\mathrm{Ohm}$ at the array edge. Figure 7 shows the elements impedances at the edge of the array, H-plane, at scan angle $\theta_{o}=60^{\circ}$. Comparing Fig. 7 with Fig. 5 it is noticed that the level of the impedance of the element at the center of the figure changes from $160 \mathrm{Ohm}$ at the array center to $125 \mathrm{Ohm}$ at the array edge.

Figure 8 shows the elements impedances in E-plane at scan angle $\theta_{\mathrm{o}}=60^{\circ}$. Comparing Fig. 8 for E-plane with Fig. 5 for H-plane it is seen that the level of impedance in Eplane is less than that of the H-plane, as expected.

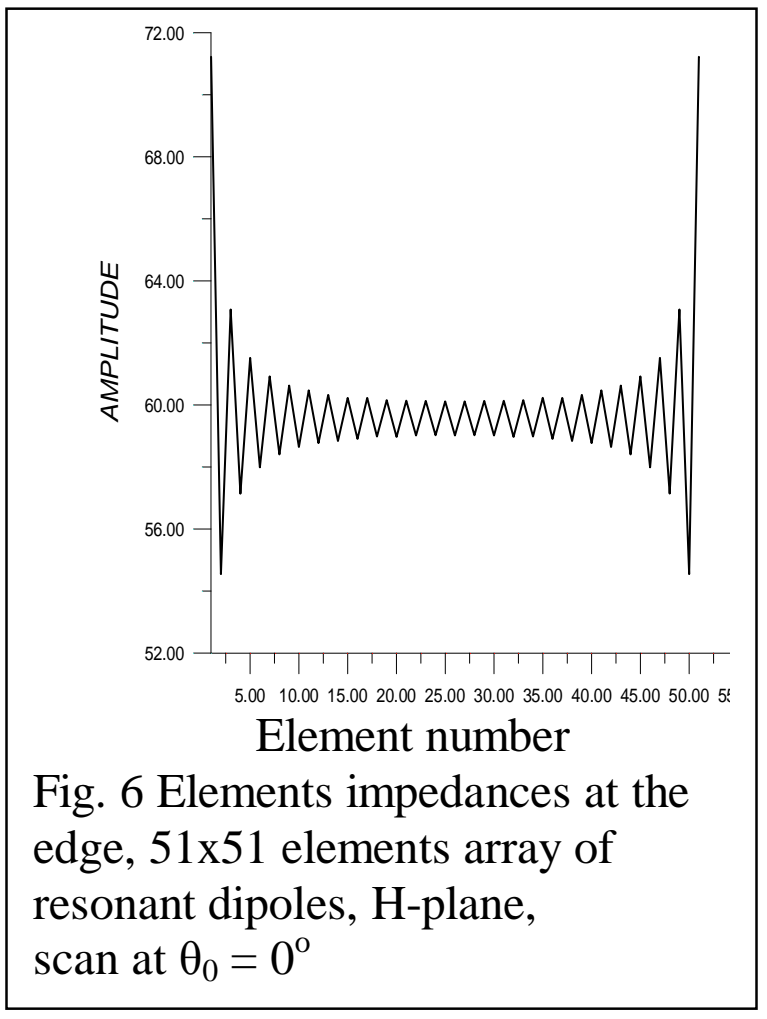

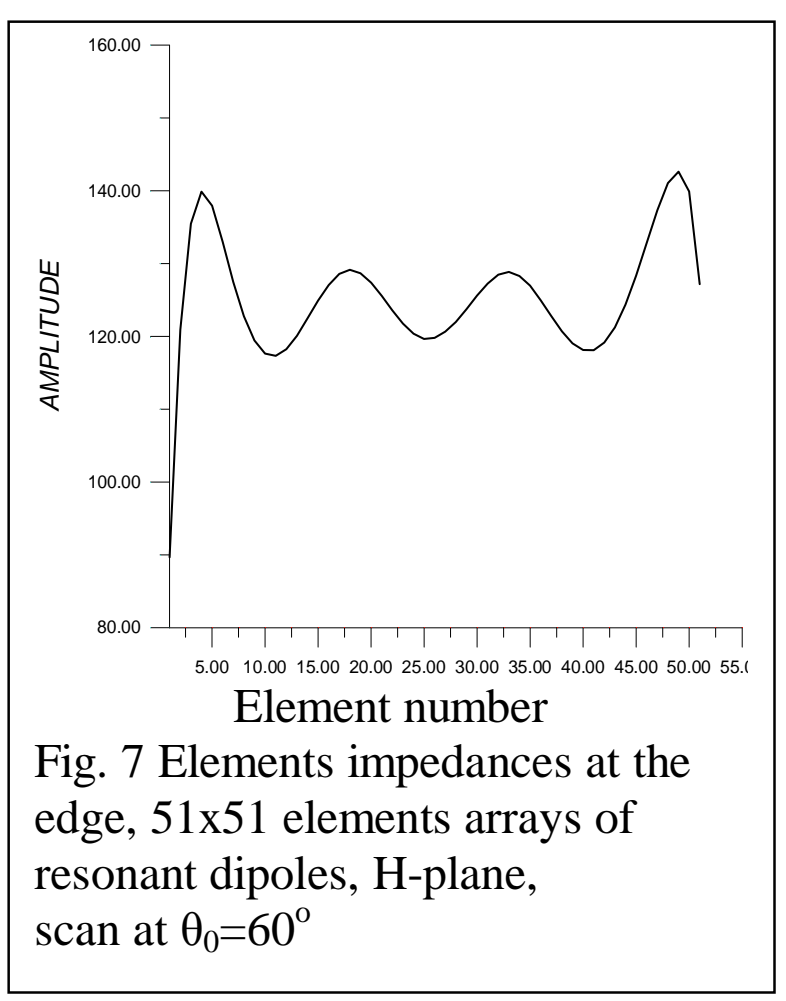




\section{Arrays with tapered excitation}

\section{Uniform excitation in $\mathbf{x}$-direction and Taylor $\bar{n}$ in $\mathbf{y}$-direction}

Figure 9 and Fig. 10 show elements impedances and the corresponding Taylor $\bar{n}$ excitation currents, respectively, at broadside scan angle $\theta_{\mathrm{o}}=0^{\circ}$ for the array in H-plane. Comparing Fig. 9 with Fig. 3 it is clear that using Taylor $\bar{n}$ distribution in y-direction decreases the level of oscillations of the impedance. Figure 11 shows the elements impedances in the array at scan angle $\theta_{o}=60^{\circ}$, in H-plane; a leaky wave is clear. Comparing Fig. 11 with Fig. 5 it is seen that as a result of tapered current in y-direction the level of the oscillation in the scan impedance is decreased and the impedance increases monotonically along the array. The latter trend can be seen in Ref. [9] with tapered excitation. Figure 12 shows elements impedances at scan angle $\theta_{\mathrm{o}}=60^{\circ}$, in $\mathrm{H}$ plane with ground plane. The leaky wave is weaker than without ground plane
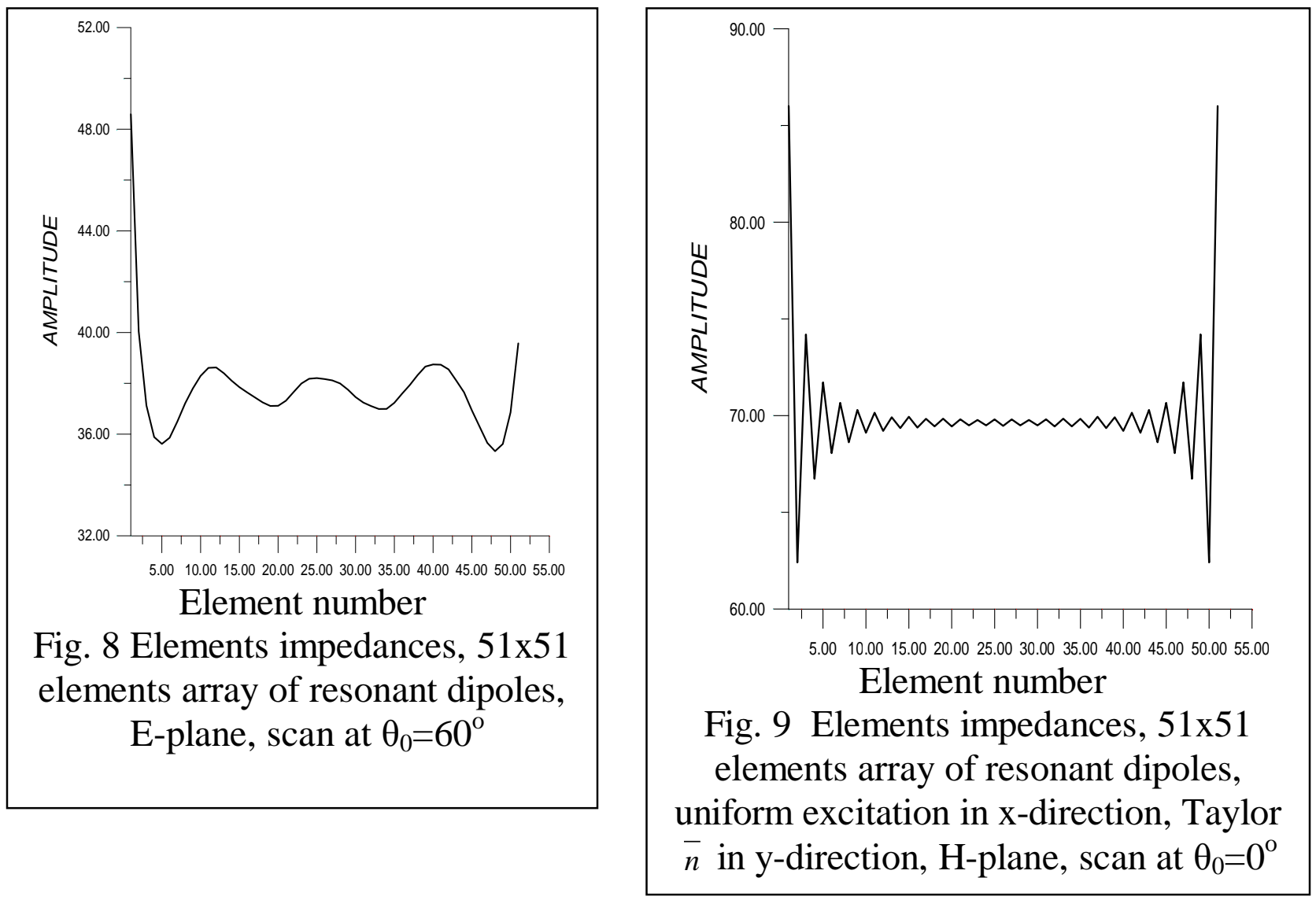

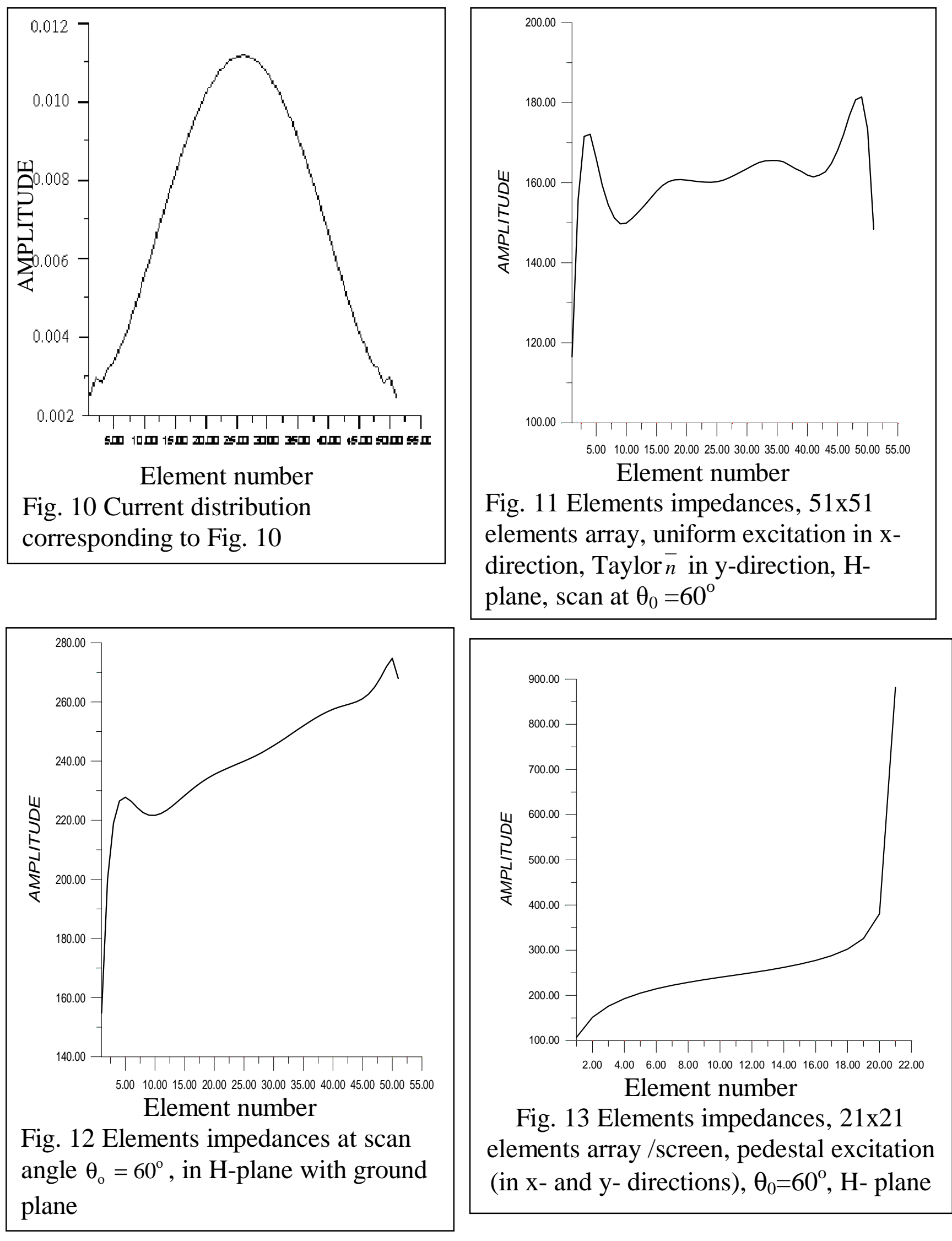


\section{Pedestal excitation in $\mathrm{x}$ - and $\mathrm{y}$ - directions}

Figure 13 and Fig. 14 show the elements impedances and the corresponding pedestal excitation current respectively for $21 \times 21$ elements array, H-plane at scan angle $\theta_{\mathrm{o}}=60^{\circ}$ with ground plane. From Fig. 13 it is clear that no leaky wave exists and the impedance increases sharply along the array. The increase of the impedance can be interpreted in view of the excited current in Fig. 14. Due to scanning, the current in the array becomes asymmetric with small deviations from the symmetric form of the exciting voltage. However, since the current near the edges is small, the change in its value, though small, but it corresponds to a large ratio w.r.t. the symmetric shape, thus it contributes to considerable variation of the impedance from that of an infinite array. This effect decreases as the elements become far from the array edge. The abnormal impedances at the array edges may be improved either by increasing the current in the edge elements, which raises the sidelobe level, or by reducing the scan angle range.

Figure 15 and 16 show the elements impedances in H-plane, broadside scan with screen on a line passing by the center of the array and on its edge, respectively. It can be seen that the impedances at the edge are slightly modulated by the pedestal excitation. This behavior is not found for uniform excitation in x-direction and Taylor $\bar{n}$ excitation in ydirection, in contrast with the pedestal excitation where tapering is in both the $\mathrm{x}$ - and $\mathrm{y}$ directions. The impedances at the array edge are lower than those at the center.
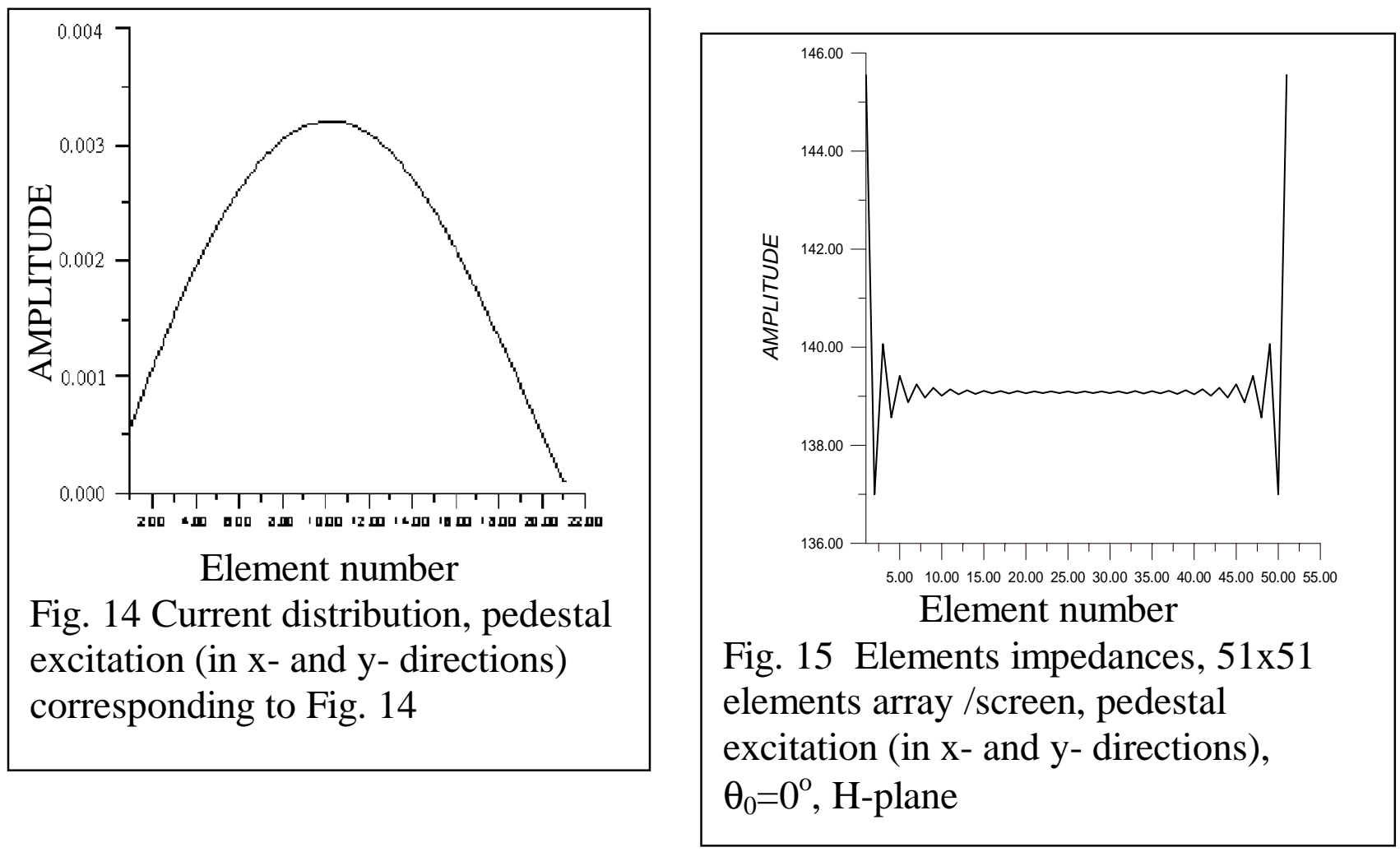

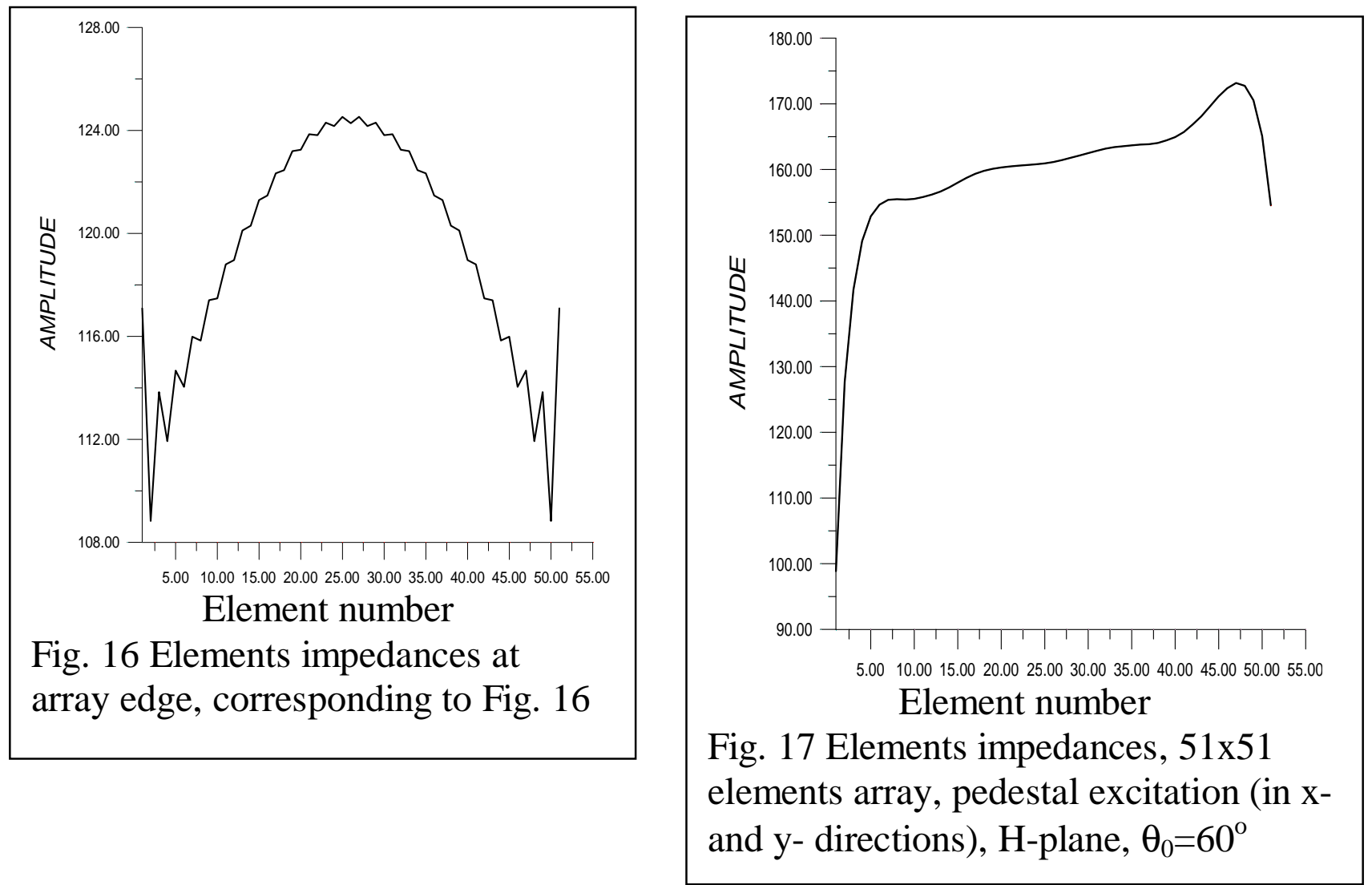

Figure 17 shows the elements impedances at scan angle $\theta_{o}=60^{\circ}, \mathrm{H}-$ plane. Comparing Fig. 17 with Fig. 5 and Fig. 11 for uniform excitation in x-direction and for Taylor $\frac{-}{n}$ excitation in the y-direction, respectively, it is clear that the leaky wave is weaker with pedestal excitation (tapering in both $\mathrm{x}$ - and $\mathrm{y}$-directions).

\section{Cosecant distribution in x-direction (fan beam) and Taylor $\bar{n}$ in y-direction}

Figure 18 shows elements impedances for 51x51 elements array, cosecant excitation in $\mathrm{x}$-direction, Taylor $\bar{n}$ in y-direction, at broadside scan $(\theta=0), \mathrm{H}$ - plane. It is clear from the figure that the average level of the impedances changes to $57 \Omega$ compared to a level of $70 \Omega$ in Fig. 9 of uniform-Taylor $\bar{n}$ excitation. This is due to the implicit different scan angles in E-plane to produce the cosecant pattern. This also leads to irregular shape of oscillations of the impedances. Figure 19 shows elements impedances at scan angle $\theta=60^{\circ}$, in H-plane. By comparing Fig. 19 with Figs. 5, 11 and 17 for uniform, Taylor $\bar{n}$ and pedestal excitations in y-direction, respectively, it is found that the impedance level decreases and the oscillations are irregular. Figures 20, 21 show elements impedances with screen at broadside scan $(\theta=0)$, H- plane, at the array center and at the edge, respectively. The impedances are more regular and higher at the array edge. Figure 22 shows elements impedances at scan angle $\theta=60^{\circ}$, in H-plane with screen. 

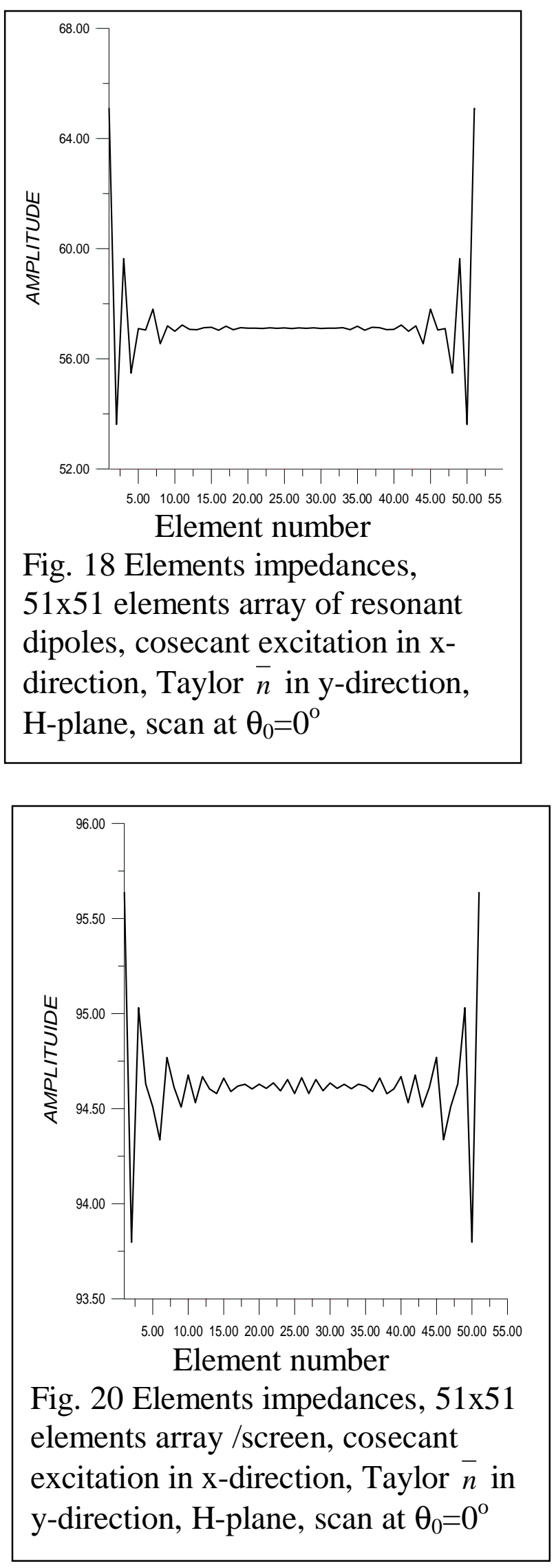
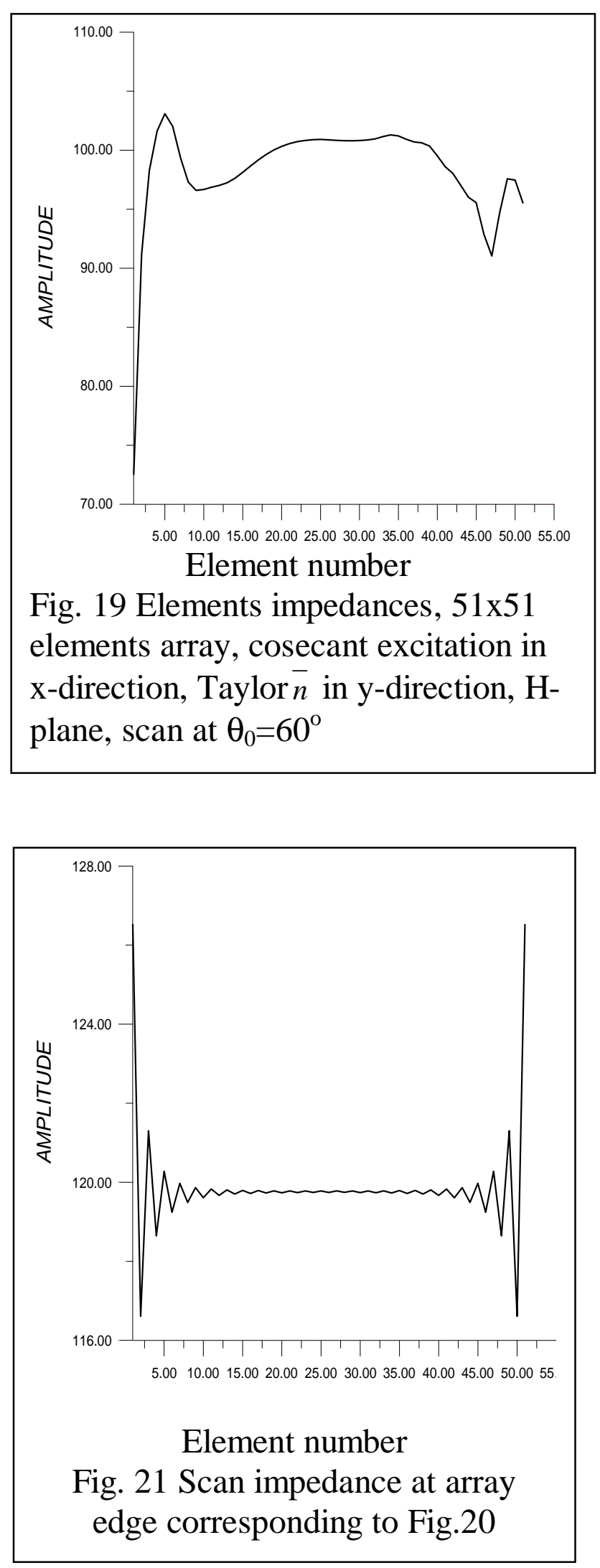


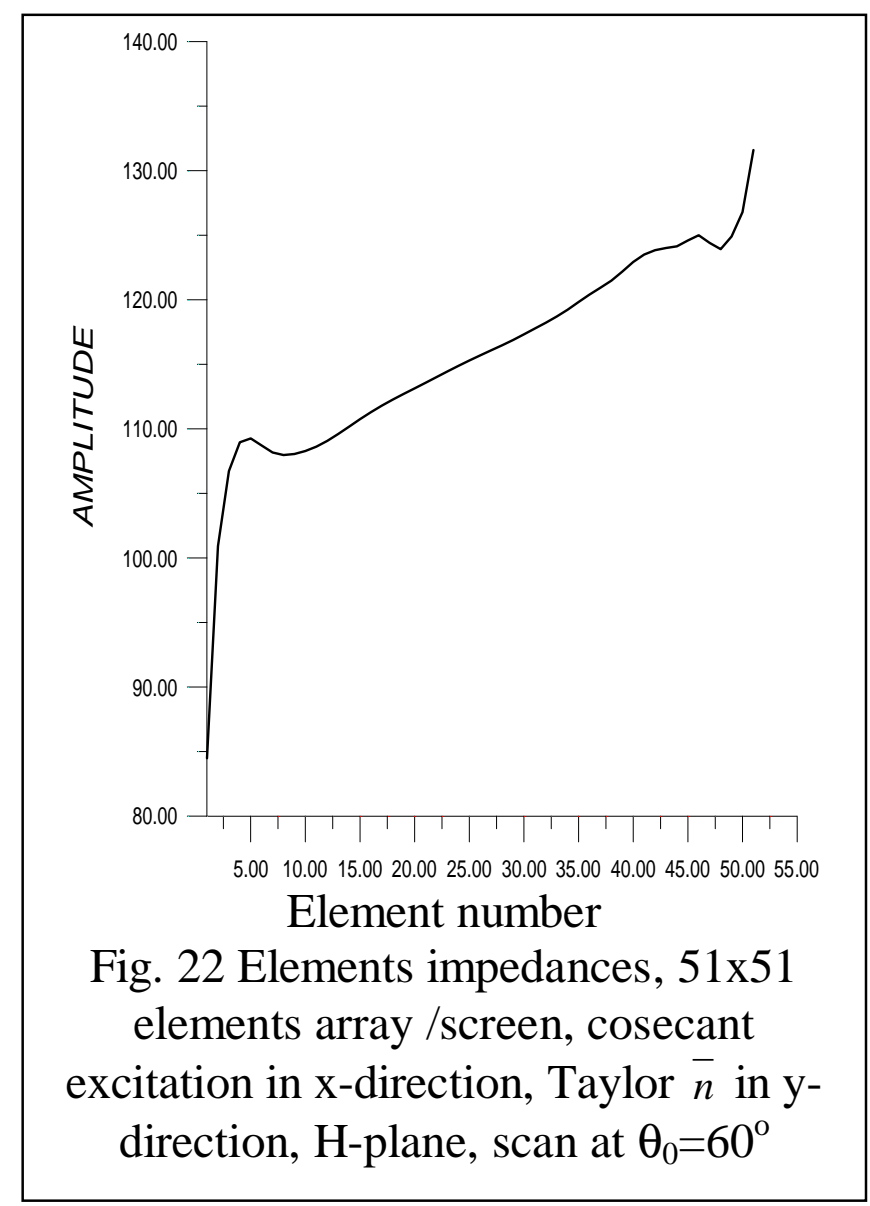

\section{Conclusions:}

The active impedances of the elements of planar finite phased arrays of flat dipoles are investigated with free excitation. The excitations investigated produce either pencil beam or fan beam radiation patterns typical for radar antennas. Cylindrical dipoles (which may be used as equivalents to strip dipoles) are considered since their mutual impedances can be accurately and fastly computed using the simple EMF method with sinusoidal current distribution, which requires a finite integration. As the scan angle increases the mutual effects increase and the effect of the self impedance decrease. In addition, the presence of the generator impedance reduces the effect of the inaccuracy in the self impedance.

It was found that with tapered excitations the current asymmetry on the array when scanning 
off broadside leads to high and low impedances at the array edges and monotonic variation of the active impedances along the array. The abnormal impedances at the array edges may be improved either by increasing the current in the edge elements, which raises the sidelobe level, or by reducing the scan angle range. With tapered excitations the leaky waves are damped, particularly for tapering in both E-and H-planes. The ground plane damps the leaky wave in the interference pattern. In the presence of scan the active impedances with different excitations are not the same due the increase of mutual effects when the beam is inclined in the presence of different current distributions, in contrast to broadside scan. The elements impedances on both edges and at corners of the array can be investigated, which can not be studied with the [published results] for finite- by- infinite arrays. 


\section{References:}

[1] R.C. Hansen," Phased Array Antennas", John Wiley, 1998

[2] A. Munk , "Finite Antenna Arrays and FSS", Wiley , 2003

[3] D. S. Janning and B. A. Munk, "Effects of surface waves on the currents of truncated periodic arrays", IEEE Trans. Antennas and Propagation, Vol.50, No. 9, Sep. 2002, pp.1254-1265.

[4] D.F. El-Hossary, E.A. El-Diwany, H.H. El-Tamaly, " Antenna Arrays and Their Applications in Electronic Warfare " , PH. D, thesis Minia University, 2007

[5] D.F. El-Hossary, E.A. El-Diwany, H.H. El-Tamaly, "surface and leaky wave propagation on planar finite dipole antenna arrays " this issue .

[6] E.C. Jordan, K. G. Balmain, "Electromagnetic Waves and Radiating Systems", Prentice Hall, 1983.

[7] C. Johnson, H. Jasic (Ed.), "Antenna Engineering Hand Book", McGraw Hill, 1984.

[8] A. Ishimaru, R.Coe, G. Miller and P.Geren, "Finite periodic structure approach to large scanning array problems" IEEE Trans. Antennas and Propagation, Vol. AP-

[9] 33, no.11, Nov.1985, pp. $1213-1220$.

K.A. Shubert and B.A. Munk, "Matching properties of arbitrarily large dielectric covered phased arrays", IEEE Trans. Antennas and Propagation, Vol. AP-31, No.1, Jan 1983, pp. 54-59 